\title{
Motivación en educación artística. Tiempo y variables
}

\author{
Inés López Manrique \\ Universidad de Oviedo
}

\section{RESUMEN}

La motivación es un factor fundamental para todo tipo de actividades, destacando en ella el papel de las emociones. La motivación está presente, tanto en la práctica artística como en el contexto educativo, clasificándose como extrínseca o intrínseca. Revisando autores fundamentales sobre motivación dirigida a estudiantes de educación artística, se encontraron variables como el tiempo, los recursos, la pasión y el ambiente. Otras estrategias consisten en valorar: la importancia de las emociones, los espacios, la experimentación, el reconocimiento del trabajo realizado, el refuerzo del sentimiento de valor y las habilidades creativas, las ideas, la introducción de nuevas herramientas, objetos inesperados y personas inesperadas. También se defiende la importancia del trabajo en equipo, salir de la zona de confort, visitar estudios de artistas o invitar a artistas a participar en clases. Se presta atención a los ritmos de cada alumno, algunos más rápidos, otros más tranquilos, dando el tiempo necesario según el ritmo de cada alumno. Hoy en día, el aumento de la digitalización también afecta a la motivación en la Educación Artística.

Palabras clave: educación artística, motivación, tiempo, variables educativas, recursos pedagógicos.

\section{RESUMEN}

Motivation is a fundamental factor for all kinds of activities, highlighting in it the role of emotions. Motivation is present, both in artistic practice and in the educational context, classifying as extrinsic or intrinsic. Reviewing fundamental authors on motivation directed to arts education students, variables such as time, resources, passion and the environment were found. Other strategies consist of evaluating: the importance of emotions, spaces, experimentation, recognition of the work done, reinforcement of the feeling of value and creative abilities, ideas, the introduction of new tools, unexpected objects and unexpected people. The importance of teamwork, getting out of the comfort zone, visiting artist studios or inviting artists to participate in classes is also defended. Attention is paid to the rhythms of each student, some faster, others calmer, giving them the necessary time according to the rhythm of each student. Today the increase in digitization also affects motivation in Art Education.

Keywords: artistic education, motivation, time, educational variables, pedagogical resources. 


\section{Motivación en educación artística}

El término motivación se utiliza constantemente en los diferentes ámbitos de la sociedad actual. En particular en el contexto educativo, se incide en que tanto el profesorado como el alumnado deben estar motivados, para así facilitar las mejores sinergias. La cuestión de la motivación en la enseñanza de las artes también suscita un creciente interés. Recientemente ha sido abordada por los especialistas de distintos ámbitos artísticos, bien de la danza (Amado, Leo, Sánchez, Sánchez y García, 2011; Carraca, 2020; GarcíaDantas, Caracuel-Tubío y Peñaloza-Gómez, 2013; Fuentes, 2007; Imbernón, 2020; Nieto, Chinchilla y Castillo, 2020), la música (Bisquerra, 2013; Carraça,2020; Martínez, 2018; Tripiana, 2018 ) o en las artes plásticas y visuales (Alsina, Mallol y Alsina, 2020; López, San Pedro, González, 2014; Molina, 2016)

En los diferentes contextos de la enseñanza, tal y como indican las leyes educativas y dictan las corrientes pedagógicas del momento, una forma de mejorar la motivación es introduciendo nuevas tecnologías y nuevas metodologías innovadoras. Respecto a las últimas es importante destacar, que en algunos casos estas "nuevas estrategias y metodologías" están integradas en las rutinas de las aulas desde hace décadas, como ocurre con el Trabajo por proyectos.

En particular, en el ámbito de las artes plásticas y visuales, algunas de las formas de trabajo que tradicionalmente eran las propias del área, ahora son etiquetadas como metodologías innovadoras. Así ocurre con la utilización de Design thinking o resolución de problemas, Visual thinking o dibujo de las ideas y procesos, desarrollo de contenidos a través de talleres, propuestas de trabajo mediante proyectos, etc.

Pero, al margen de metodologías y corrientes educativas, nos preguntamos ¿es la motivación la misma en todas las situaciones educativas? ¿qué mueve a las personas de diferentes épocas y entornos a expresarse plásticamente?, ¿que esperan conseguir con esta actividad? ¿qué les motiva en el aprendizaje de las artes plásticas y visuales?

Los estudios que han abordado el tema de la motivación, la vinculan siempre al autoconcepto (González y Tourón, 1992) y las emociones (Damasio,1999) e insisten en que las emociones tienen el poder de motivarnos. A su vez las artes facilitan emociones de carácter positivo (Calderón, Gustems-Carnicer, Martín-Piñol, Fuentes-Moreno y Portela-Fontán, 2020), aunque esta vinculación será diferente según la modalidad de arte, el género, la edad y la formación o experiencia de las personas.

Según la clasificación habitual de motivación en el contexto educativo, existe una motivación extrínseca y otra intrínseca. La primera tiene relación con la obtención de resultados externos y la segunda no supone ganancias externas para el individuo. Por lo tanto en la práctica de las actividades artísticas y en la educación artística podríamos considerar la motivación de dos tipos. En el primer caso, de la motivación extrínseca, se espera la obtención de algo externo como podría ser resolver una tarea pedida por el profesorado al alumnado.También se hablaría de motivación extrínseca cuando se esperan "recompensas" externas a la persona, como alcanzar una calificación en la asignatura, conseguir una compensación económica a través de un concurso artístico o un trabajo, recibir reconocimiento social a través de las actividades artísticas, etc.

Respecto a la motivación intrínseca, en ella no habría recompensa externa, sino los aspectos internos que son los que suponen el motor y satisfacción de la situación. En el caso de las artes plásticas y visuales como motivaciones internas se pueden considerar: canalización de la expresión y creatividad, realización de un ejercicio de libertad personal, que contengan fines terapéutico, potenciación y refinamiento de la técnica artística, mejora de la socialización con los compañeros y compañeras de clase, interés por la ampliación de los conocimientos artísticos y su aprendizaje, etc.

Pero, es importante tener en cuenta que dentro de una misma persona, según el momento vital en que se encuentre unas tendrán más peso que otras e irán variando, sin haber necesariamente un equilibrio entre las motivaciones intrínsecas y extrínsecas.

Es ahí cuando la motivación se convierte en un verdadero reto para el alumnado y el profesorado.

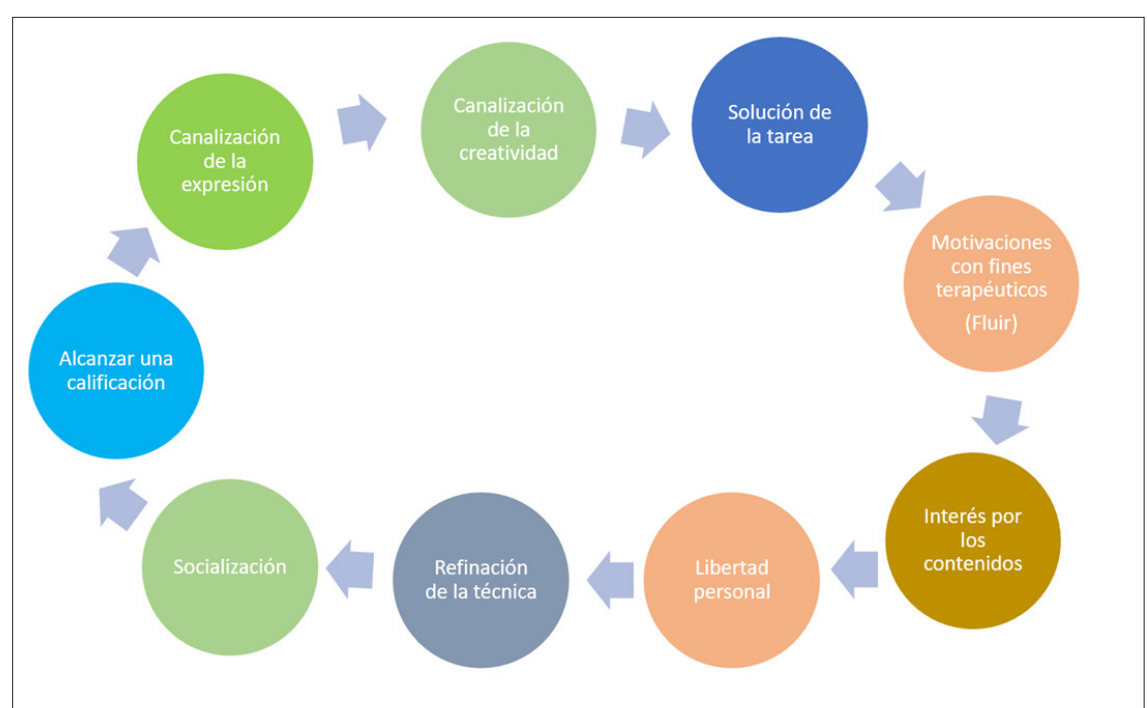

Figura 1. Diagrama sobre posibles motivaciones en el aprendizaje de las artes plásticas y visuales. Imagen: elaboración propia. 


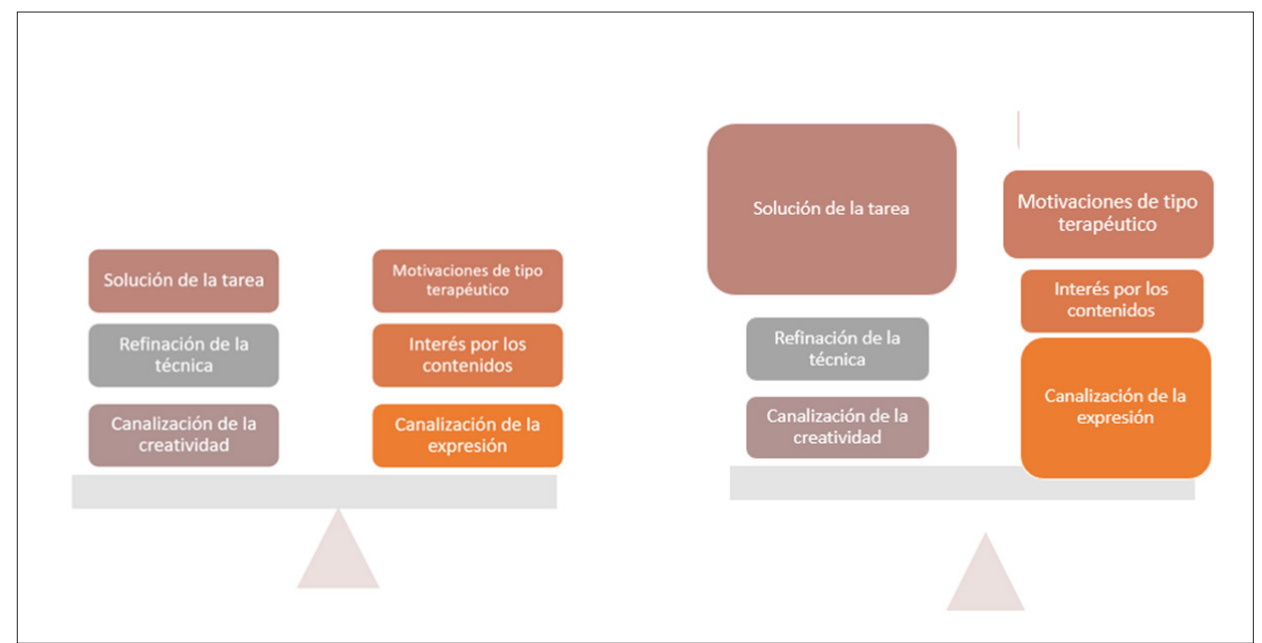

Figura 2. Diferentes motivaciones y su peso en una persona. Imagen: elaboración propia.

\section{Variables y motivación en el aprendizaje de las artes plásticas y visuales}

En el caso de los estudios profesionales de enseñanzas artísticas parece que el factor motivacional principal es el interés y apego a la materia (Martín y Martínez, 2002). Pero esto no impide que intervengan también otros aspectos relativos a la motivación en la educación artística.

Las variables que median en los procesos de creación y enseñanza-aprendizaje de las artes plásticas, son diversas y en algunos casos escurridizas.

Los diferentes niveles educativos y edades son muy variados. La edad es una de ellas, que siempre estará presente. Aunque esa edad y la maduración no se encuentren necesariamente relacionadas. Los expertos emiten recomendaciones en torno a grupos concretos de edades.

Morales (2001), Pavlou (2006) y Lekue (2010), en estudios sobre motivación en niños, preadolescentes y adolescentes, determinaron que la motivación disminuye con el aumento de la edad, siendo más elevada en las niñas que en los niños. Parece ser que la crisis del dibujo de la preadolescencia se refleja en este tipo de investigaciones, ya que la baja autoevaluación respecto a la materia aumentaría con los años.

En conclusión, tanto género como edad se pueden convertir en factores muy determinantes de la motivación hacia las materias del ámbito de la educación visual y plástica.

Por otra parte, para Marco $(1992,1997)$, en los preadolescentes y adolescentes el grupo social será clave, así como aquellos materiales y técnicas artísticas que se utilicen. Esta autora subraya además la importancia de un buen clima de atención y escucha por parte del docente y el resto del alumnado. Porque para el individuo, sentirse escuchado por los integrantes del grupo y recibir un feedback sobre su obra por medio de comentarios será de gran valía.

Sin duda, para esto se precisa de espacio y tiempo. Pasando a ser este momento de diálogo y escucha otro "proceso dentro del proceso", que finalmente se convierte en estímulo para la persona. De esta manera se podrá reforzar la sensación de autocapacitación del estudiante, sobre todo a partir de la adolescencia. Por lo que se sugiere momentos en las sesiones de trabajo destinados a la conversación y escucha, donde se produzca un intercambio respetuoso de opiniones, que favorezca la percepción de competencia en las tareas.

También, en esta franja de edad, un factor motivante será el encontrar la figura de un profesor o profesora con un amplio número de recursos y conocimientos. De igual manera, y ya en adultos jóvenes, disfrutar con las tareas y obtener una buena calificación académica influirá en la motivación de los estudiantes de las asignaturas del ámbito de la educación artística (López, San Pedro y González, 2014).

Pero, en ocasiones, el trepidante ritmo de vida y el exceso de tareas no permiten detenerse con suficiente cuidado en crear esos momentos de atención y escucha, que debidamente guiados pueden reconfortar y motivar tanto. Como si la vida fuera un balancín en el que hacer equilibrios, introducir momentos de pausa y revisión es motivador. $\mathrm{E}$ igualmente, crear pequeñas sorpresas y giros inesperados de la programación temporal, ayudarán a centrar de nuevo la atención.

Por otra parte, González y Maeso (2005) destacaron algunas directrices para favorecer la motivación en el ámbito de las personas mayores. Resaltando la introducción de cambios que supongan sorpresa o "el salir de la zona de confort" como una de ellas. Pero de nuevo insisten en lo beneficioso de la comunicación horizontal y el refuerzo positivo. Por lo tanto, hay cuestiones, como la escucha y la empatía, que conectan al individuo con la educación artística más allá de los niveles educativos, géneros y edades.

Así mismo, para las personas, los espacios son importantes impulsadores de la motivación, la creatividad e inspiración (Marco,1997; Esquinas y Sánchez, 2011; Nuere, 2011; López, 2019; Montiel, Mayoral, Navarro, Maiques y Marco, 2020). Los cuales, pueden favorecer a la organización de las materias e influir en la relación de los integrantes del grupo. Los ambientes educativos deberían ser lugares cuidados, diseñados según pautas ergonómicas, que incluyan una adecuada: iluminación, dimensiones, sonido, materiales, 
temperatura, color, etc. Al describirlo como "adecuado" nos referimos también a su versatilidad, ya que han de permitir el trabajo en función de diferentes actividades, zonas, dinámicas, y con ello también de diferentes tiempos. Afortunadamente, las tareas en educación artística son muy variadas, lo cual se hace especialmente evidente al desarrollar trabajos con técnicas que incluyan proyectar, diferentes fases, pruebas, necesidades técnicas de secadofijado, repeticiones, etc. Es decir, donde de nuevo el factor tiempo y su previsión son importantes.

Es indudable que nos adentramos en una nueva era donde la tecnologización será constante. También la tecnología es un elemento que se propone para motivar al alumnado de las materias artísticas, siendo la realidad aumentada y la programación herramientas didácticas poderosas (Avilés, Mirete y Maquilón, 2020; Regis, 2020; Sáez-López y Sevillano-García,2017).

\section{El tiempo y la personalidad como variables}

Como se señalaba al inicio de la disertación, el profesorado del área además de las propuestas de trabajo con nuevas metodologías de enseñanza y el uso de herramientas tecnológicas, cuenta con sencillas pero grandes estrategias metodológicas. Algunas de ellas facilitan el desarrollo pedagógico y la motivación en las aulas. En la medida de lo posible los y las docentes las hemos intentado incorporar en nuestras clases, algunas de ellas son: incorporar referentes motivadores, utilizar elementos que sirvan como detonantes de las acciones, acercamiento a la obra de arte o contar con ella presencialmente en el aula, trabajo grupal, revisión de libros y documentos gráficos con el grupo, acudir a lugares externos para la realización de las actividades, incluir visitas a museos y galerías, audición musical, inclusión de visitas de personas externas ligadas al arte o a los proyectos que se trabajan, realizar lecturas evocadoras, visionar películas y vídeos, provocar tormentas de ideas y preguntas al aire, intervenir el aula, o propuestas con un objeto o una actividad fuera de contexto, etc.

Sin duda, son muchas las posibilidades y recursos con los que contamos en la educación artística. La naturaleza de la materia permite

Figura 3. Diferentes estrategias metodológicas utilizadas para motivar: visita de un artista al aula, trabajo con materiales nuevos, utilización de nuevas tecnologías en trabajo grupal y propuestas fotográficas basadas en artistas reconocidos (proyecto del alumno Marco Mas). Imagen: elaboración propia. su integración en la práctica habitual de las clases y con ello facilita el desarrollo de las capacidades e influir en las motivaciones (intrínsecas y extrínsecas) del alumnado.

Para el incremento y trabajo de la motivación será necesario establecer una determinada duración o tiempo de las actividades y procesos, que faciliten transcurrir de una intensidad a otra y que serán diferentes según las circunstancias.

El tiempo, comprendido de una forma amplia, es un elemento inherente a todos los procesos artísticos y aprendizajes, así como un actor protagonista en la evolución madurativa (Han, 2005). Esto lleva a pensar que las coordenadas espacio-tiempo están presentes en cualquier proceso. Desde el ámbito de las Ciencias Sociales, Trepat y Comes (1998) comentan que el tiempo sí se puede vivir, pero no se puede representar. Este segundo aspecto varía en el arte, ya que tiene esa capacidad de plasmación de lo objetivo-subjetivo, tangible-intangible que le confiere su infinitud.

Quizás el reflexionar con el alumnado sobre estas cuestiones sirva para conocer su comportamiento ante las ideas de tiempo y temporalidad.

Se comentaba anteriormente que jugar con los espacios puede ser una fuente de motivación y consideramos que participar de la variable tiempo también es una estrategia para potenciarla. En este sentido, cada persona es en sí misma un sistema que tiene su propia velocidad y tiempo, los cuales estarán marcados por su personalidad y circunstancias. Sin duda habrá quienes prefieran realizar
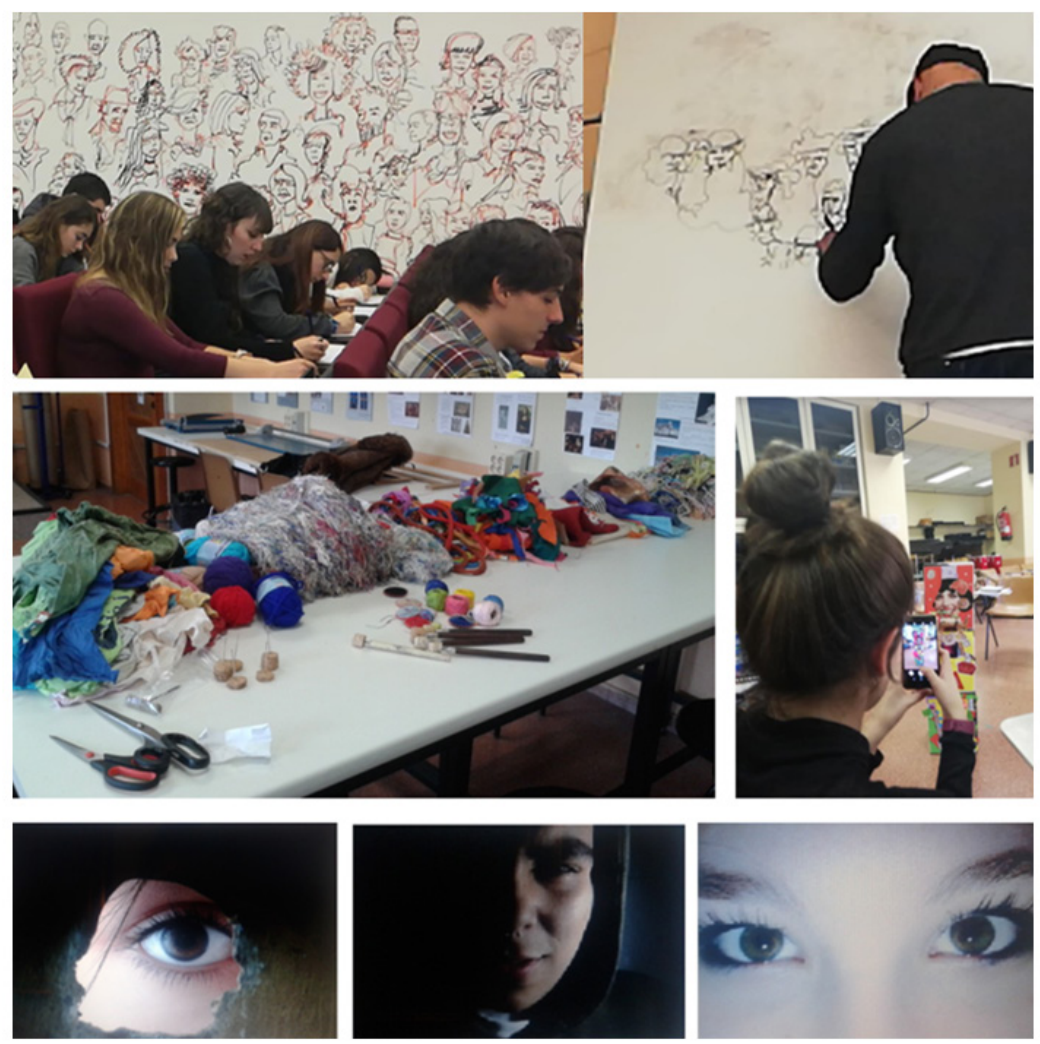
tareas con calma, con reflexión, en lejanía respecto al mundo de las pantallas y de la inmediatez que nos rodea. Mientras que otros individuos necesitarán una aceleración, un ritmo más rápido de trabajo sin dilaciones.

Puede hacerse partícipe al alumnado de la idea que sugiere Han (2005) sobre lo erróneo de creer que la aceleración del tiempo supondrá aceleración de las opciones y obtención de la plenitud. Sin necesariamente decantarse por la tendencia slow, en las artes plásticas y visuales la teoría del fluir según cada ser, su propia individualidad y circunstancias encajaría mejor en un aula donde se presentan variedad de personas y personalidades.

Por lo que una estrategia fundamental para cualquier docente será, siempre que sea posible, el trabajo con un grupo pequeño que permita conocer bien las características psicológicas, necesidades, motivaciones, fortalezas y debilidades de cada uno de los alumnos y alumnas. Su diversidad nos obliga a tener en cuenta que lo temporal en cada uno será diferente, convirtiéndose en otra herramienta más para motivarles. Aunque también se encontrarán casos en que las personas tendrán una motivación interna muy marcada y no necesitarán tanto ese empuje o estrategia del profesorado. Pero sin duda, el carácter de la persona juega un papel importante aquí. La Marca (2007, p.122) define la personalidad como "una serie de cualidades psíquicas heredadas y adquiridas; una síntesis de las actividades internas del ser humano y de su experiencia exterior que se constituye sobre la base temperamental y del carácter. La personalidad es el resultado de factores temperamentales $y$ caracteriológicos que durante el desarrollo de la persona y bajo la acción del ambiente exterior configuran al individuo, sobre todo en lo psíquico"

A su vez, Quintana (1995) describió el carácter psicológico como la forma que tiene el individuo de participar en el mundo según la utilización de sus facultades. Abarcando esto lo que define su existencia y le diferencia de los demás, es decir su sentir, obrar, objetivos, valoraciones, decisiones y juicios.

Lo cierto es que las clasificaciones básicas nos dicen que una persona con un carácter flemático podrá desarrollar las tareas de una forma más calmada que una sanguínea o una colérica.

Quizás haya personas con una psicología natural que no necesiten de estudios y teorías psicológicas, pero las clasificaciones de la personalidad y temperamentos del ser humano pueden ser útiles para aquel no versado en esa materia y programar acciones y tiempos según esas variables.

\section{Conclusiones}

El tiempo es un factor a tener en cuenta en las artes plásticas y visuales y su docencia, por lo que destinar atención a esta cuestión e incluirla como variable motivacional, puede ser beneficioso. Dentro de estructuras ordenadas también hay disincronías positivas en algunas personas.

Para concluir, señalar que el cuidado de la motivación en la práctica docente produce resultados positivos en los y las estudiantes, lo cual revierte también positivamente el profesorado. En este sentido, Torrés y Juanola (1998) al repasar las estrategias para la motivación en niños comentan que el papel de los docentes puede llegar a ser el de un mago con trucos de todo tipo, donde la satisfacción de enseñar es la mejor de las motivaciones. Se destaca a continuación una síntesis de las propuestas de Eisner (2004) para motivar al profesorado. De nuevo se sugiere el cuidado del entorno en que se van a desarrollar las actividades artísticas, la ubicación de los estudiantes, los materiales y la interacción de los estudiantes. También el mantener una actitud de observación, así como una postura crítica ante las propias acciones educativas parece ser una buena estrategia. Finalmente, impulsar la pasión del propio docente como el mejor motor para crear esa energía de ilusión, interés y amor por la educación artística.

\section{Referencias bibliográficas}

Alsina, M., Mallol, C., \& Alsina, A. (2020). Currículum competencial y educación artística en secundaria. Resultados de una experiencia de cocreación basada en el ABP/Competence curriculum and artistic education in secon-dary. Results of a co-creation experience based on ABP. ARTSEDUCA, (26), 104-117.

Amado, D., Leo, F. M., Sánchez, P. A., Sánchez, D., \& García, T. (2011). Interacción de la teoría de la autodeterminación en la fluidez disposicional en practicantes de danza. Cuadernos de Psicología del Deporte, 11(1), 7-17.

Avilés, M., Mirete, A. B., \& Maquilón, J. J. (2020, March). Innovación educativa basada en realidad aumentada: Una experiencia en el área de dibujo. In Congreso Internacional de Investigación e innovación en educación infantil y primaria.

Bisquerra, A. (2013). Música, emoción y motivación: una experiencia interdisciplinaria. In Educación emocional: propuestas para educadores y familias (pp. 187-204). Bilbao: Desclée de Brouwer.

Calderón, D., Gustems-Carnicer, J., Martín-Piñol, C., Fuentes-Moreno, C., \& Portela-Fontán, A. (2020). Emociones en la experiencia artística: claves para el desarrollo educativo y social/Emotions in the artistic experience: keys to educational and social development. ARTSEDUCA, (25), 85-101.

Carraça, E. V. (2020). Efeitos psicológicos da música em praticantes de exercício: Uma revisão sistemática. Cuadernos de Psicología del Deporte, 20(2), 152-173.https://doi.org/10.6018/cpd.368961

Damasio, A.R. (1999). The feeling of What Happens: Body and Emotion in The Making of Conciousness. Londres: Heinemann.

Esquinas, F. y Sánchez, M. ( 2011). Didáctica del Dibujo: artes plásticas y visuales. Barcelona: Ministerio de Educación-Graó.

García-Dantas, A., Caracuel-Tubío, J. C., \& Peñaloza-Gómez, R. (2013). Intervención formativa con el profesorado de danza e influencia motivacional en su alumnado. Cuadernos de Psicología del Deporte, 13(2), 9-20. 
González, M.C. y Tourón, J. (1992). Autoconcepto y rendimiento escolar. Sus implicaciones en la motivación y en la autoregulación del aprendizaje. Pamplona: EUNSA.

González, M.R. y Maeso, F. (2005). El valor de la motivación en la educación artística con personas mayores. Arte, Individuo y Sociedad, 17, 43-60.

Fuentes, S. (2007). Motivación para bailarines. Bilbao: Danza Getxo.

Han, B. C. (2015). El aroma del tiempo: un ensayo filosófico sobre el arte de demorarse. Barcelona: Herder.

Imbernón, S. (2020). Psicología y danza profesional, dos disciplinas conocidas aún por encontrarse. Danzaratte: Revista del Conservatorio Superior de Danza de Málaga, (13), 22-37.

La Marca, A. (2007). Educación personalizada y formación del carácter. Estudios sobre Educación, 13, 113-131.

Lekue, P. (2010). Características psicoinstruccionales del alumnado preadolescente y rendimiento académico en la Educación Artística escolar. Universidad del País Vasco.

López, I., San Pedro, J. C., \& González, C. (2014). La motivación en el área de Expresión Plástica. Arte, Individuo y Sociedad, 26(2), 199-213.

López, S. (2018). Esencia: diseño de espacios educativos: aprendizaje y creatividad. Madrid: Khaf.

Marco, P. (1992). Educación Artística y preadolescencia. Revista Interuniversitaria de Formación del Profesorado, 24,99-111.

Marco, P. (1997). Motivación y creatividad en la preadolescencia. Valladolid: Universidad de Vallodolid.

Martín, J. y Martínez, R. (2002). Diseño, aplicación y resultados de una encuesta para evaluar aspectos motivacionales en la Escuela Municipal de Música y Danza de San Sebastián. Ponencia II Simposio de la Asociación de Motivación y Emoción. Salamanca, 2002.

Martínez, I. (2018). Motivación y emoción en música.: Estado de la cuestión y aportaciones para la innovación educativa. Artseduca, (19), 62-83.

Molina, S. (2016). Learning By Doing. Cómo fomentar el interés y la motivación en el alumnado de Educación Plástica y Visual por el tema de las axonometrías. In Tecnología, innovación e investigación en los procesos de enseñanza-aprendizaje (pp. 917-926). Octaedro.

Montiel, I., Mayoral, A. M., Navarro Pedreño, J., Maiques, S., \& Marco, G. (2020). Linking Sustainable Development Goals with Thermal Comfort and Lighting Conditions in Educational Environments. Education Sciences, 10(3), 65 .

Morales, J.J. (2001). La evaluación en el área de Educación Plástica en la Educación Secundaria Obligatoria. Tesis doctoral. Barcelona: Universidad Autónoma de Barcelona.

Nieto, M. M., Chinchilla, J. L., \& Castillo, A. (2020). Estudio de los procesos cognitivos en bailarines semi-profesionales. Retos: nuevas tendencias en educación física, deporte y recreación, (37), 493-497.

Nuere, S. (2011). La organización del aula: espacio de interacción y comunicación,27-45. En Esquinas y Sánchez, Didáctica del dibujo: artes plásticas y visuales (2011). Barcelona: Ministerio de Educación-Graó.

Pavlou, V. (2006). Pre-adolescent's perceptions of competence, motivation and engagement in art activities. Journal of Art Design Education, 25 (2), 194-204.
Trepat, C. y Comes, P. (1998). El tiempo y el espacio en la didáctica de las ciencias sociales. Barcelona: Graó.

Quintana, J.M. (1995). Pedagogía psicológica: la educación del carácter y de la personalidad. Madrid: Dykinson.

Sáez-López, J. M., \& Sevillano-García, M. L. (2017). Sensors, programming and devices in Art Education sessions. One case in the context of primary education /Sensores, programación y dispositivos en sesiones de Educación Artística. Un caso en el contexto de Educación Primaria. Cultura y educación, 29(2), 350-384.

Regis, P. J. (2020). Ponencias: Arte social y otras Terapias creativas.-El uso de las redes sociales como medio educativo para el conocimiento del arte en el contexto de la educación no formal. In I Congreso internacional de intervención psicosocial, arte social y arteterápia.

Torrés, M. y Juanola, R. (1998). Dibujar, mirar y pensar. Consideraciones sobre educación artística. Barcelona: Rosa Sensat.

Tripiana, S. (2018). Eficacia de la motivación en la enseñanza de la música instrumental. Valoración comparativa y modelos subyacentes inferidos de la bibliografía de didáctica del piano del siglo XX. Ars Educa, 24, 1-14. DOI: http://dx.doi.org/10.6035/Artseduca.2019.24.4 